\title{
Éditorial
}

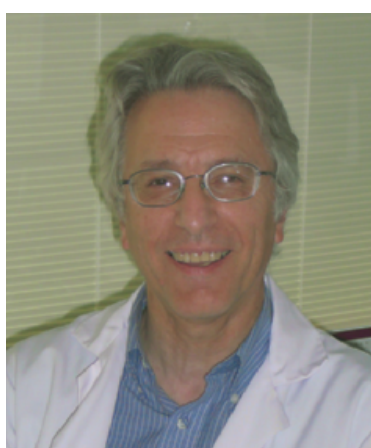

Pierre Marti Rédacteur en chef adjoint

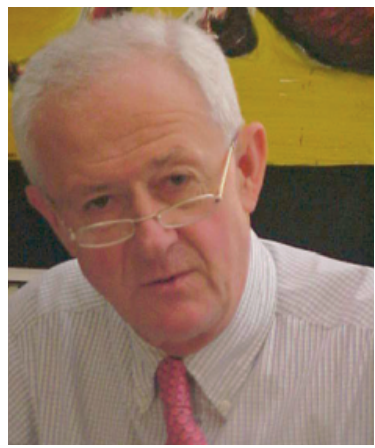

Daniel Serin Rédacteur en chef

Toute l'équipe d'Oncomagazine souhaite la bienvenue à Jeanne qui vient agrandir la famille d'Émilie Gillet !

\section{C'est une grande folie que de vouloir être sage tout seul}

\section{La Rochefoucauld}

\section{Ami lecteur,}

Les connaissances concernant la cancérogenèse, la génétique et le développement des tumeurs augmentent à une vitesse telle qu'il devient difficile pour le clinicien de les intégrer. Mais au-delà de la maladie cancer, c'est une personne qui est malade avec ses angoisses, ses craintes, son histoire personnelle et familiale mais aussi sa sensibilité aux traitements elle-même conditionnée par son patrimoine génétique, etc. Il en résulte l'impérieuse nécessité de passer du " prêt à porter » au " sur mesure ».

Vous retrouverez dans les « Actus » relatives à l'ASCO 2010 des informations qui n'ont pas été sous le feu des projecteurs mais n'en constituent pas moins des éléments potentiellement utiles à votre pratique. En revenant d'Eurocancer, F. Ellien et D. Delfieu s'intéressent à l'image du corps dans les cancers ORL et aux traitements antalgiques, deux thématiques qui concernent au plus haut point le malade.

Seule la recherche permettra d'améliorer les traitements. Le reportage concernant I'unité cancer et développement du Centre Léon Bérard, l'essai LIBER relatif à la place de la chirurgie, entre fatalisme et interventionnisme chez les patientes BCRA1/2, le programme PROCHE visant à accroître la sécurité des patients traités par chimiothérapie constituent trois aspects de cette recherche.

S'il est indispensable pour les cancérologues d'actualiser leurs connaissances et de participer aux essais cliniques, les patients n'en tireront bénéfice que sous réserve que les médicaments soient disponibles et que des établissements de qualité existent. L'article de Martine Poivre traite des entrées et sorties des médicaments sur la liste des produits « hors GHS » et le reportage sur la clinique Pasteur de Toulouse illustre ce qu'est aujourd'hui un établissement privé de haute qualité.

Mais tout cela ne suffit pas, il faut s'efforcer de «prendre du recul». Dominique Gros traite du cas de Frances Burney et son vécu de la chirurgie mammaire qu'elle a subie (subir est bien le mot) alors que Jacques Rouëssé traite de l'évolution des idées en Occident sur le diagnostic du cancer et sur la carcinogenèse. Ces deux articles nous rappellent que tout médecin doit rester modeste quant à ce qu'il sait et que « rien n'est jamais acquis à I'homme ».

L'une des plus grandes modifications dans l'exercice de la médecine réside probablement dans la place croissante du patient et dans l'abandon d'un paternalisme infantilisant au profit d'une relation de type adulte - adulte. Les patients ont à nous dire et nous avons à écouter et entendre. Le résumé du colloque d'Europa Donna ou le « pas coupable mais responsable » de La Maison du Cancer témoignent de ce dire.

La multiplicité des approches, la diversité des éléments de prise en charge peuvent conduire à une cacophonie contre productive. Véronique Trillet Lenoir, présidente du Conseil National de Cancérologie, nous explique en quoi la coordination des efforts est nécessaire et quel rôle unificateur peut jouer le Conseil National de Cancérologie.

Bonne lecture. •

Pierre Marti, Daniel Serin

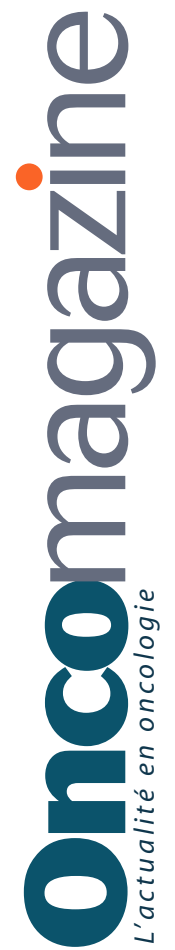

